\title{
The Eddy-current-induced Head-tail Instability at the KEK-PS
}

\author{
T. Toyama, D. Arakawa, S. Igarashi, J. Kishiro, E. Nakamura and K. Takayama \\ High Energy Accelerator Research Organization (KEK) \\ 1-1 Oho, Tsukuba, Ibaraki 305 Japan
}

\begin{abstract}
The head-tail instability of the $12 \mathrm{GeV}$ PS at KEK, has been confirmed by extensive experiments. It turns out that the eddy-current-induced sextupole fields in the rectangularshaped vacuum pipe in the main bending magnets played a crucial role in the head-tail instability during acceleration. The eddy current effect was verified by field measurement and chromaticity measurement in a way of different fieldrampings. The coupling impedances of the present injection kickers and new fast-extraction (FX) kickers, the dominant impedance sources, have been examined on the test bench. The growth rate is calculated.
\end{abstract}

\section{INTRODUCTION}

The head-tail instability limited the beam intensity of the $12 \mathrm{GeV}$ PS at KEK[1]. The eddy-current-induced sextupole field in the vacuum pipe in the main bending magnets forces the horizontal chromaticity into shifting from negative to positive value during acceleration. Then longitudinal modes $\ell=0,1$ and 2 become unstable. Especially the mode $\ell=0$ is fatal.

The chromaticity change is proportional to $(d B / d t) / B$ if it comes from eddy current. In the KEK-PS MR $d B / d t$ saturates at $2.3 \mathrm{~T} / \mathrm{s}$, while $B$ increases as the beam is accelerated. Therefore the chromaticity change is larger for lower energy. That is why the instability occurs just after acceleration start.

Organization of the present paper is as follows. First, the eddy current in the vacuum pipes in the bending magnets is manifested to be a main source that causes the positive chromaticity. Second, the result of impedance measurement of injection and FX kickers is given. The observed growth rate before and after the installation of the FX kickers are briefly described.

\section{EDDY-CURRENT-INDUCED SEXTUPOLE FIELD}

The sextupole fields induced by the eddy current propotional to $d B / d t$ is considered to cause a change in the chromaticity. Its size was measured for two different $d B / d t$ patterns. One is $d B / d t=2.3[T / s] \times t /(100[m s])$ for 0 $<\mathrm{t}<100 \mathrm{~ms}$, i.e. $100 \mathrm{~ms}$ smoothing pattern. The other is $d B / d t=2.3[T / s] \times t /(200[m s])$ for $0<\mathrm{t}<200 \mathrm{~ms}$, i.e. $200 \mathrm{~ms}$ smoothing pattern. The calculated and measured chromaticities during a beginning part of acceleration are plotted in Fig.1 and Fig.2, respectively. Solid line and dashed line in Fig.1 indicate the case of the $100 \mathrm{~ms}$ and $200 \mathrm{~ms}$ smoothing pattern, respectively. Filled circles and open circles in Fig. 2 indicate the case of the $100 \mathrm{~ms}$ and the $200 \mathrm{~ms}$ smoothing pattern, respectively. Global feature in the measured temporal variation of $\xi_{x}$ is qualitatively and quantitatively similar to the calculated one except an unknown $\xi_{x}$ shift of 1-2, which has been observed for these years.

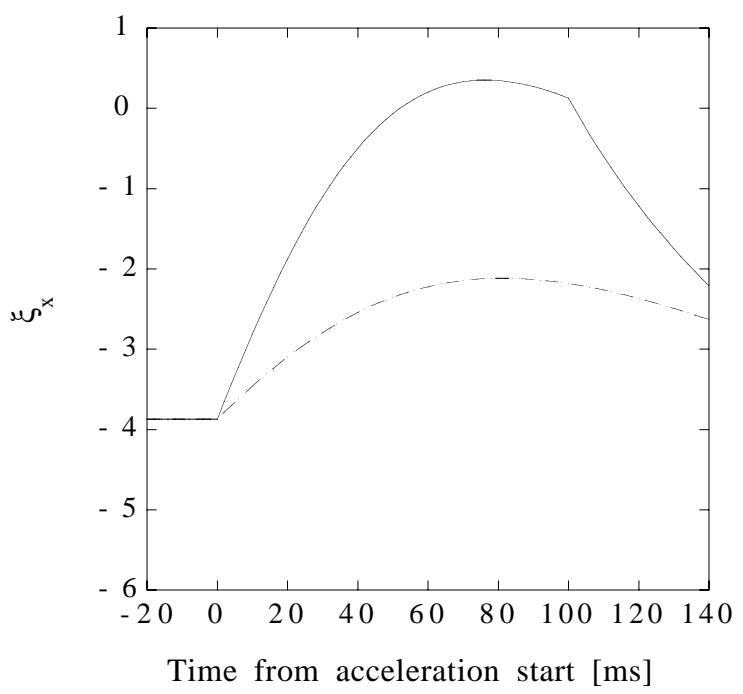

Figure 1: Calculated horizontal chromaticity

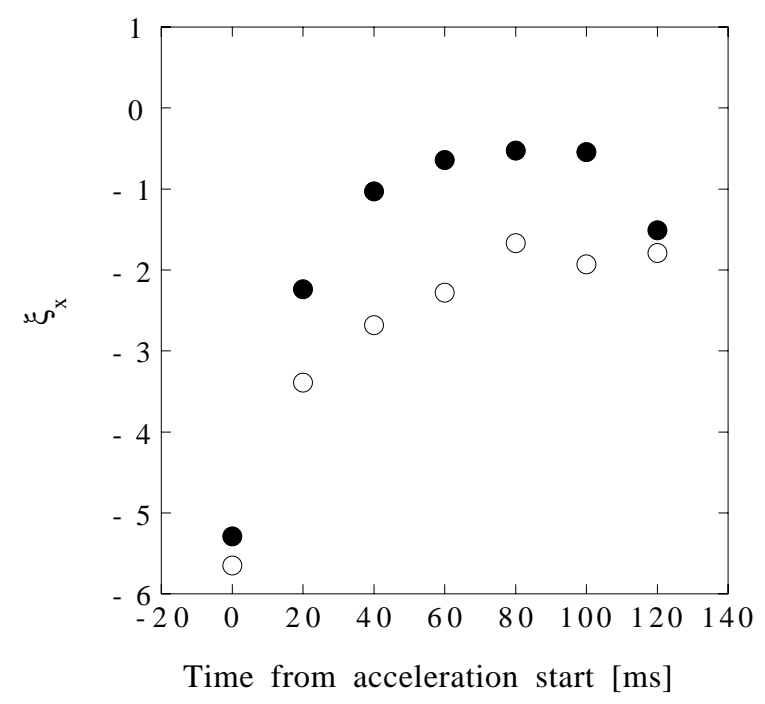

Figure 2: Measured horizontal chromaticity

The eddy-current-induced sextupole field component was directly measured in a case with/without a model pipe 
of SUS316L in the monitor bending magnet connected with main bending magnets in series. "Gaussmeter"(LakeShore 460) consisting of Hall probes and processing units was employed for the measurement. Two Hall probes were placed almost at the center in the longitudinal direction of the magnet. One probe locating at a fixed position was used as a reference. The output voltage excursion of the other probe from the reference signal is recorded by using time domain data acquisition of a 16-bit FFT analyzer (ADVANTEST R9211C SERVO ANALYZER). The signal was averaged over a few tens of field pattern cycles. It was essential to use a difference signal of two probes to maximize the ADC signal-to-noise-ratio and to cancel an undesirable long-term field variation. The frequency band width of the measurement system was sufficient for our purpose.

The difference between the measured fields with and without the vacuum pipe is taken. In Fig. 3 a field variation along the horizontal axis at the timing of $100 \mathrm{~ms}$ from the beginning of acceleration is indicated by open circles. The field variation at the center is set to be zero for convenience.

It is in good agreement with the calculations using three analytic formulae: a two-parallel-plate approximation ( dashed line )[2], a numerical integration with a realistic shape ( solid line )[3]. A 2D simulation by the boundary element method for eddy-current-induced fields also gives a consistent result[4].

Consequently, the change in the chromaticity is attributed to the eddy-current-induced sextupole fields.

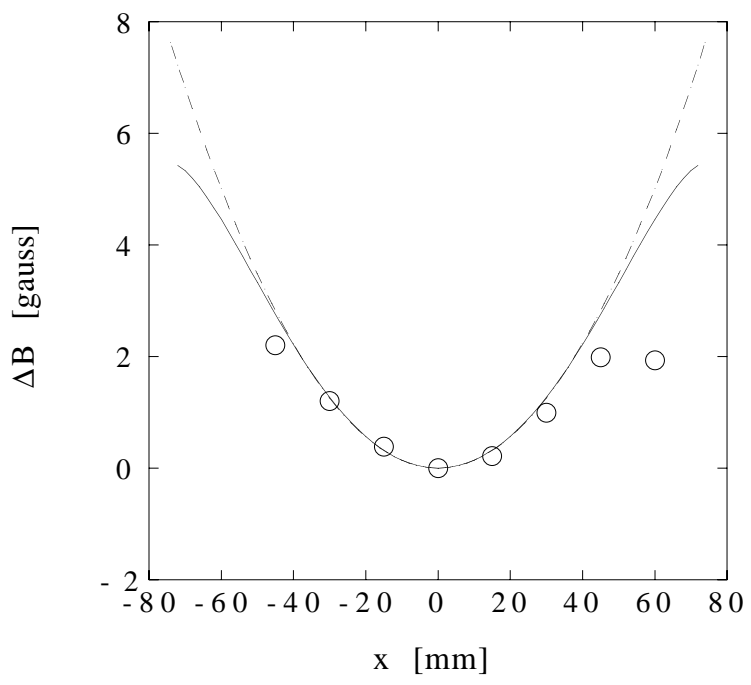

Figure 3: Field variation due to eddy current in the vacuum pipe

\section{KICKER IMPEDANCES}

The growth rate measurement has suggested that the main sources of the coupling impedances are kickers and resistive wall[1]. It was urgent to evaluate the coupling impedance of newly installed FX kickers[5] for longbaseline neutrino oscillation experiment.
To evaluate the kicker coupling impedance, both of theoretical and experimental approach have been employed. The real part of the transverse coupling impedances of the injection and FX kickers were calculated by using the standard theory of travelling wave kickers[6],

- C-type

$$
R e\left[Z_{T}\right] \approx \frac{c}{\omega} \frac{Z_{0}}{4 b^{2}} \eta,
$$

- "Twin c-type"[5]

$$
R e\left[Z_{T}\right] \approx \frac{c}{\omega} \frac{Z_{0}}{b^{2}} \eta,
$$

where $c$ is the velocity of light, $Z_{0}$ the characteristic impedance of the kicker, $\omega$ the angular frequency, $b$ the gap width and $\eta$ the factor depending on the cable connection and termination of the kicker.

The measurement was performed using a copper wire of $1 \mathrm{~mm}$ in diameter. At first $S_{21}$ in " $\mathrm{S}$ " matrix was measured

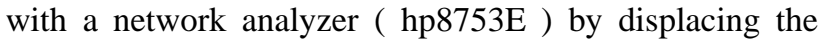
wire in the horizontal direction. The $S_{21}$ component was translated to the $Z_{T}$ by using the formula for distributed impedance[7].

The results are plotted in Fig.4 for the injection kicker and in Fig.5 for seven FX kickers. In the figures dots indicate the measured value and solid line the calculated one. Due to the limitation of the experimental setup, the terminations of the kickers are not same as to the practical setup. In the present measurement, the matched loads were connected with the injection kicker at both ends. The FX kicker is conneted with a matched load at one end and short-circuited at the other end. The disagreement between the calculated and measured $\operatorname{Re}\left[Z_{T}\right]$ for both type of kickers may come from incomplete error correction or ferrite loss which were not included in the calculations. They are subjects to be solved in future.

The additional impedance newly introduced by the seven FX kickers seems to be much larger than that of the five injection kickers for the frequency range of our interest.

In addition, the longitudinal impedance measurement of the FX kickers embedded in the large rectangular chamber implied sharp resonances in a few hundred $\mathrm{MHz}$ region as shown by the dashed line in Fig.6. It is potentially dangerous for the microwave instability serious at transition crossing[8]. It seems to be caused by the gaps between kicker modules. Then the gaps between kickers were filled up by the short copper pipes of the similar inner size. It reduced the impedance peak height by a factor of two to four as indicated by the solid line in Fig.6.

\section{CONCLUSION}

The growth rate which is calculated by introducing the coupling impedances of the resistive wall and kickers into Sacherer's formula[9] should become to be large after installing the FX kickers. The growth rate measurements 


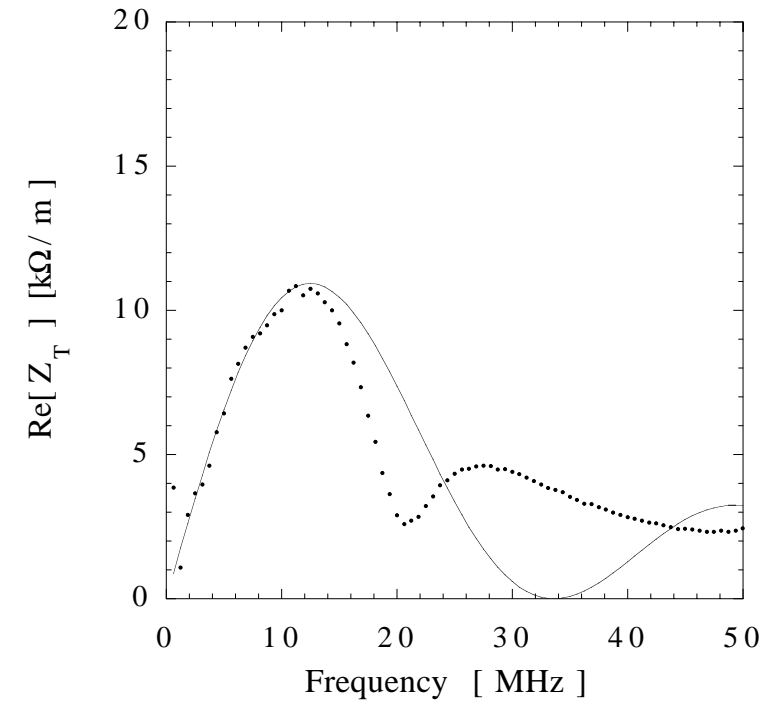

Figure 4: Coupling impedance of one injection kicker

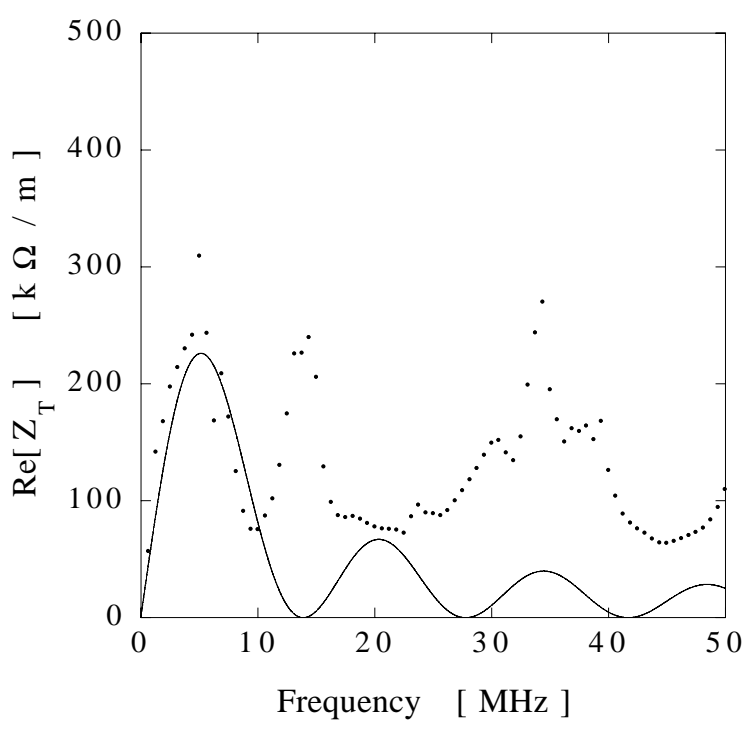

Figure 5: Coupling impedance of seven FX kickers

have been performed during a commissioning run for the long-baseline neutrino oscillation experiment. The growth rate has increased by about a factor of two after installing the FX kickers under certain conditions. Although this can be reproduced by the calculation, a full agreement in the overall behaviour has not been achieved yet.

Octupole magnets have been re-located with four-fold symmetry so as to minimize a dynamic aperture reduction. Landau damping effect with the octupoles was crucial for the operation after installing the FX kickers not only during acceleration but also during the injection flat bottom.

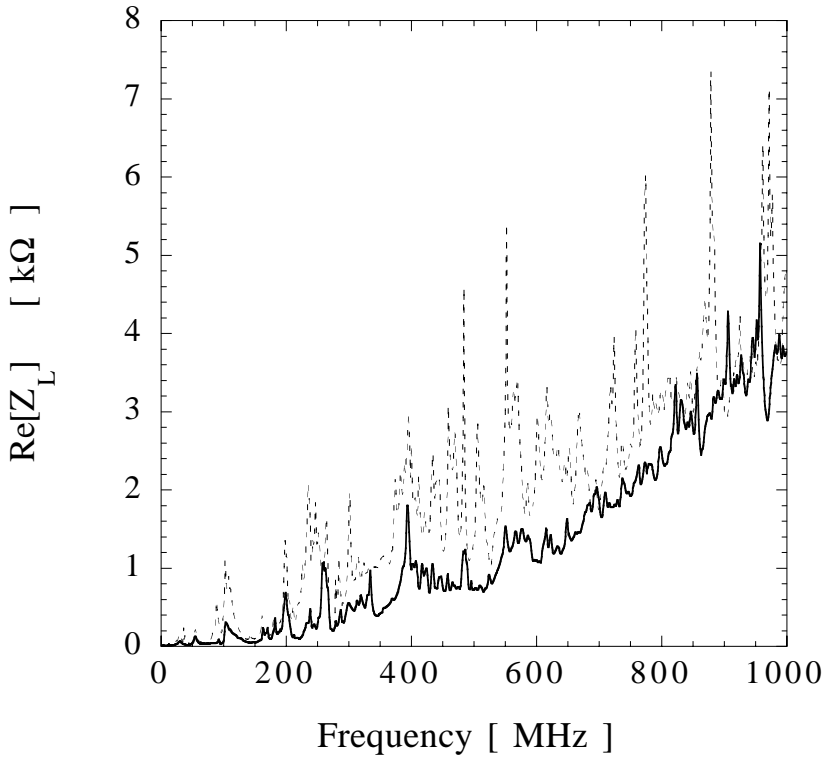

Figure 6: Coupling impedance of seven FX kickers

\section{ACKNOWLEDGEMENTS}

M. Shirakata assisted us in the experiment with a beam. Y. Kanai and Y. Shimosaki contributed to field measurement. F. Caspers gave useful comments on the coupling impedance measurement. M. Uota assisted in measuring the coupling impedance. W. Chou called our attension to the work of S. Y. Lee. M. Kumada suggested chromaticity measurement with different $d B / d t$ 's and readily accepted our request to use his gaussmeter.

\section{REFERENCES}

[1] T. Toyama, D. Arakawa, S. Igarashi, J. Kishiro, K. Koba and K. Takayama, Proc. of the 1997 Part. Accel. Conf. (1997) 1599. T. Toyama, D. Arakawa, S. Igarashi, J. Kishiro and K. Takayama, Proc. of the first Asian Part. Accel. Conf. (1998) 471.

[2] D. A. Edwards and M. J. Syphers, "An Introduction to the Physics of High Energy Accelerators", John Wiley \& Sons, Inc., 1993.

[3] S.Y. Lee, Nucl. Instrum. Methods A300 (1991) 151.

[4] T. Toyama, to be published.

[5] T. Kawakubo, S. Murasugi, E. Nakamura and S. Tazawa, Proc. of the first Asian Part. Accel. Conf. (1998) 582.

[6] H. Hahn and A. Ratti, AD/RHIC/RD-111, 1997.

[7] L.S. Walling, D.E. McMurry, D.V. Neuffer and H.A. Thiessen, N.I.M. A281 (1989) 433.

[8] K. Takayama, D. Arakawa, J. Kishiro, K. Koba and M. Yoshii, Phys. Rev. Lett. 78 (1997) 871. K. Takayama, D. Arakawa, J. Kishiro, K. Koba, T. Toyama and M. Yoshii,Proc. of the 1997 Part. Accel. Conf. (1997) 1548.

[9] A. Chao, "Physics of collective beam instabilities in high energy accelerators", John Wiley \& Sons, Inc., 1993 and the references there in. 